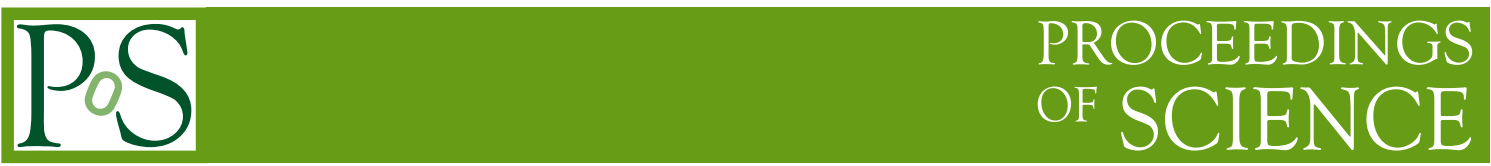

\title{
Search for eV Sterile Neutrinos - The STEREO Experiment
}

\section{Mathieu Vialat ${ }^{a, *}$}

${ }^{a}$ Institut Laue-Langevin, CS 0156,

71 Avenue des Martyrs, 38000 Grenoble, France

E-mail: vialatm@ill.fr

\begin{abstract}
STEREO is a high precision experiment studying the antineutrinos coming from the core, highly enriched in ${ }^{235} \mathrm{U}$, of the nuclear reactor of the Institut Laue-Langevin (Grenoble,France). The aim is to investigate two anomalies revealed by the measurements of several reactor neutrino experiments. The first one is linked to the absolute flux coming from a reactor ("Reactor Antineutrino Anomaly [RAA]") and could be explained by the existence of a new oscillation at short baseline to a light sterile neutrino. The second anomaly is related to the shape of the antineutrino energy spectrum where an excess of events compared to the prediction has been measured around $5 \mathrm{MeV}$ ("5 MeV Bump"). Here, preliminary results of the test for a new oscillation using 334(543) days reactor ON(OFF), which constitutes the entire dataset of the STEREO experiment, will be reported. They are compatible with the no-oscillation hypothesis and reject the RAA best-fit at more than $4 \sigma$. Concerning the analysis of the energy spectrum shape, the results of the analysis of 273(519) days ON(OFF) show a confirmation of the events excess between 5 and $6 \mathrm{MeV}$.
\end{abstract}

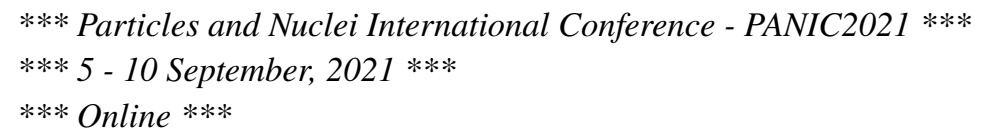

${ }^{*}$ Speaker 


\section{Introduction}

The Reactor Antineutrino Anomaly (RAA) was established in 2011 with a new prediction of the reactor antineutrino spectrum wich has shown a $6 \%$ deficit of the average of rates measured by previous reactor experiments [1]. Moreover the antineutrino energy spectra measured by several low enrichment reactor experiments revealed an anomaly of the spectral shape with an excess of events around $5 \mathrm{MeV}$ compared to the prediction. Even if the combination of these two anomalies seems to point to a bias in the prediction, possibly due to underestimated systematics, the RAA could also be explained by the introduction of a fourth, sterile neutrino at the $\mathrm{eV}$-scale mass involving an oscillation of electronic antineutrinos at short distance.

In a model with only one sterile neutrino, one can write the probability of disappearance of the $\overline{v_{e}}$ at short baseline as:

$$
P_{\overline{v_{e}} \rightarrow \overline{v_{e}}} \approx 1-\sin ^{2}\left(2 \theta_{e e}\right) \sin ^{2}\left(1.27 \frac{\Delta m_{41}^{2}\left[\mathrm{eV}^{2}\right] L[\mathrm{~m}]}{E_{\overline{v_{e}}}[\mathrm{MeV}]}\right)
$$

where $\theta_{e e}$ is the mixing angle, $\Delta m_{41}^{2}$ is the difference of masses square of the mass eigenstates and $L$ and $E_{\overline{v_{e}}}$ are respectively the distance of propagation and the energy of the antineutrino.

Experiments investigating the RAA, like STEREO, aim to measure the relative distortion in shape of the neutrino energy spectra measured at different distances from the reactor core in order to be sensitive to an oscillation without being impacted by a possible bias in the prediction.

\section{Description of the STEREO experiment}

ILL reactor The STEREO detector was located at $10 \mathrm{~m}$ from the core of the reactor of the Institut-Laue-Langevin (ILL) in Grenoble, France. It has a nominal power of $\approx 58 \mathrm{MW}_{\text {th }}$. The advantages of this reactor are the small size of its core $(\varnothing 40 \mathrm{~cm} \times 80 \mathrm{~cm})$ which gives a high resolution on the distance of propagation and the enrichment of the core in ${ }^{235} \mathrm{U}$ which allows to approximate that neutrinos come entirely from the products of the fission of this isotope $\mathbf{9 9 . 7 \%}$ of the neutrino flux). This last point is important for the shape analysis and the determination of the contribution of ${ }^{235} \mathrm{U}$ fission products to the Bump at $5 \mathrm{MeV}$. However, the ILL being a research reactor, the experiments placed around STEREO can create neutron and gamma background which are reduced by the installation of shieldings around the detector. STEREO is also rather sensitive, as a surface level experiment, to the cosmological background and that's why the detector has been placed under a water chanel providing a 15 m.w.e. protection.

Neutrino detection and detector design The antineutrinos are detected by their interactions with hydrogen via Inverse Beta Decay (IBD): $\overline{v_{e}}+p \rightarrow n+e^{+}$. The IBD events are signed by the coïncidence between a prompt event created by the practically instantaneous deposition of energy and annihilation of the positron, and a delayed event coming from the capture of the neutron by gadolinium $(\mathrm{Gd})$ atoms. The energy of the antineutrino is directly related to the one of the prompt event by the relation $E_{\overline{v_{e}}}=E_{e^{+}}+0.782$.

The detector was designed to detect the IBD events in a target volume of $2 \mathrm{~m}^{3}$ divided in 6 detection cells filled with liquid scintillator doped with Gd. A second volume, filled with non doped 
liquid scintillator, surrounds the target to detect the $\gamma$-rays escaping from the main volume. It is called gamma-catcher. The scintillation light is reflected on the walls of the cells to be detected on top by 48 PMTs distributed over each target and gamma-catcher cell. Homogeneous light collection and optical contact are made by acrylic buffers containing mineral oil. The figure 1 shows the different parts of the detector.

Data taking The STEREO detector was dismantled in november 2020 after 4 years of data taking, divided in 3 periods of acquisitions visible in figure 2.

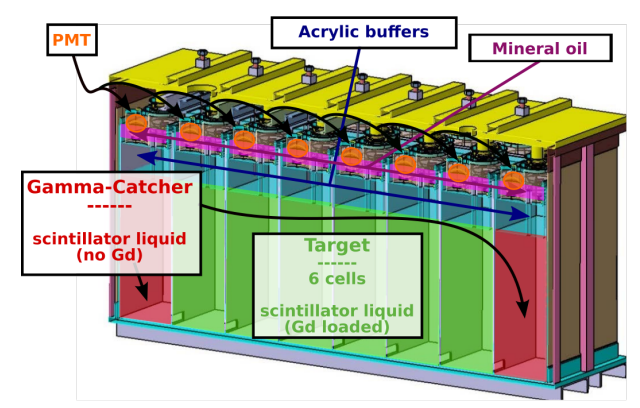

Figure 1: Drawing of the STEREO detector.

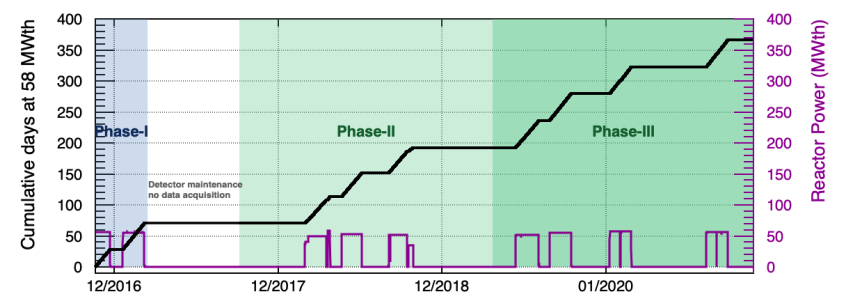

Figure 2: Reactor power in function of the date. The 3 phases of data taking and the number of ON cumulated days are presented.

\section{Detector response}

A daily and automatic monitoring of the electronic and liquid stability is performed using LEDs with dedicated acquisition. The monitoring of the energy response is done using a set of $\gamma$ and neutron sources placed inside and around the detector and the energy deposition for each event is reconstructed thanks to an algorithm taking into account light cross-talk between cells. The systematic uncertainties on reconstructed energy are evaluated by comparing the reconstructed energy for the different sources in $\mathrm{MC}$ and data. Furthermore a spectrum of the beta decay of ${ }^{12} \mathrm{~B}$ is studied and a global fit over sources and ${ }^{12} \mathrm{~B}$ gives uncertainties in the data/MC ratio inferior to $1 \%$ over the expected neutrino energy range. The time stability is monitored by the evolution of the n-H capture peak. A sub-percent stabilty $(\approx 0.3 \%)$ is achieved.

\section{Antineutrino signal extraction}

The selection of the IBD events is made by looking at the coincidence between prompt and delayed signals within a $70 \mu$ s time window. A set of other cuts is then used to minimise the background events while keeping a high neutrino detection efficiency. Details of the IBD selection are given in reference [2].

Pulse Shape Discrimination (PSD) is used to handle the remaining background. It separates the prompt events into two populations: electron-recoil, like the $\overline{v_{e}}$ events, and proton-recoil, induced by fast neutrons mainly created by the interaction of cosmic muons in the detector shielding. The difference of pulse shape is due to different scintillation decay times depending on the nature of the energy deposition. The observable used in STEREO is the ratio $Q_{\text {tail }} / Q_{t o t}$ where $Q_{\text {tail }}$ is the charge in the tail of the pulse and $Q_{t o t}$ the total charge. The $\overline{v_{e}}$ rates are extracted, for each cell and 
energy bin separately, by a fit of PSD distributions acquired in reactor-ON using a model based on reactor-OFF data. A scaling factor is used to compensate the difference of normalization between $\mathrm{ON}$ and OFF due to different acquisition times but also variations of atmospheric pressure or level of water in the water channel above STEREO. The neutrino component is fitted by a gaussian function and the rate for each bin and cell is given by its integral. However, in order to apply this method, the PSD shape has to be stable over the data taking period. An evolution of the PSD has been measured and a correction, taking into account time and temperature evolution, is applied before the fitting procedure to insure the stability in time. The spectra extracted can then be analyzed.

\section{Shape Analysis}

The STEREO experiment aims to provide a pure ${ }^{235} \mathrm{U}$ spectrum in antineutrino energy to be compared with predictions and other experiments. The method used is based on the unfolding of the measured energy spectrum $D$ into the antineutrino energy spectrum $\phi$ through the detector response matrix $R$ expressed by the formula:

$$
\chi^{2}(\phi)=(R(\alpha) \phi-D)^{T} V_{\text {stat }}^{-1}(R(\alpha) \phi-D)+|\alpha|+r \cdot \boldsymbol{\Lambda}(\boldsymbol{\phi})
$$

where the $\alpha$ parameters modelize the systematic uncertainties. $r \cdot \boldsymbol{\Lambda}(\phi)$ is a regularization term to avoid high fluctuation between two successive energy bins. The parameter $r$ is tuned to limit the dependence to the prior shape used for the regularization, here the Huber model. This method uses nuisance parameters to take into account the systematic uncertainties but another method has also been developed using total covariance matrix. More details on the methods can be found in reference [3].

The Phase-II and III data have been jointly analyzed with this. The antineutrino energy spectrum obtained is given on figure 3 where it is compared to the Huber (HM) and Summation (SM) models (see reference [3]). The agreement with HM is $\chi^{2} / \mathrm{ndf}=40.5 / 17$ when comparing only the shape with the area normalized spectrum. Fitting a potential excess of events around $5-6 \mathrm{MeV}$ gives a significatively better agreement $\chi^{2} / \mathrm{ndf}=11.6 / 14$. The best-fit bump amplitude is $0.107 \pm 0.021$ for a mean position at $5.39 \pm 0.13 \mathrm{MeV}$. This result confirms the excess of events of approximately $10 \%$ measured previously by experiments at reactors with low ${ }^{235} \mathrm{U}$ enrichment.

\section{Oscillation analysis}

The oscillation analysis is performed by comparing the variations of the antineutrino spectra between cells. The spectra are compared by this formula:

$$
\begin{aligned}
\chi^{2} & =\sum_{l=1}^{N_{\text {cells }}} \sum_{i=1}^{N_{\text {Ebins }}}\left(\frac{D_{l, i}-\varphi_{i} M_{l, i}\left(\sin ^{2}\left(2 \theta_{e e}\right), \Delta m_{41}^{2}, \alpha\right)}{\sigma_{l, i}}\right)^{2}+\sum_{l=1}^{N_{\text {cells }}}\left(\frac{\alpha_{l}^{\text {EscaleU }}}{\sigma_{l}^{\text {EscaleU }}}\right)^{2} \\
& +\left(\frac{\alpha^{\text {EscaleC }}}{\sigma^{\text {EscaleC }}}\right)^{2}+\sum_{l=1}^{N_{\text {cells }}}\left(\frac{\alpha_{l}^{\text {NormU }}}{\sigma_{l}^{\text {NormU }}}\right)^{2}+\sum_{l=1}^{N_{\text {cells }}}\left(\frac{\alpha_{l}^{\text {ReacBkg }}}{1}\right)^{2}
\end{aligned}
$$




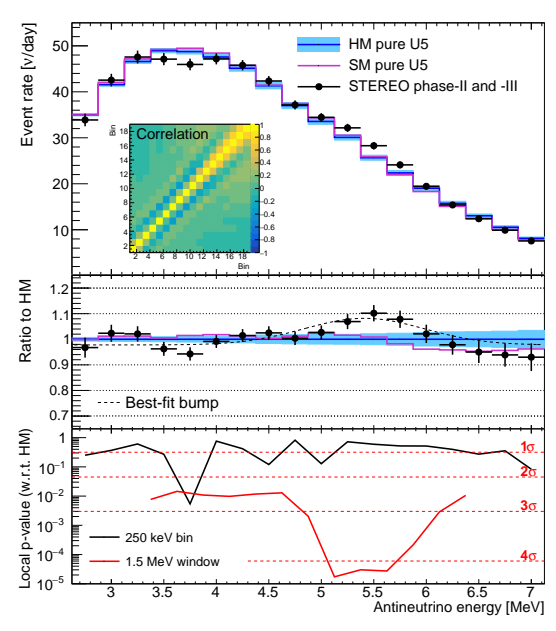

Figure 3: (top) Energy spectrum compared to the Huber (HM) and Summation (SM) models normalised to the surface of the measured spectrum. The non-trivial correlation matrix is displayed. indicated by the gray line and the black star while (middle) Ratios to HM prediction. (bottom) local the expected sensitivity is shown in blue. p-value calculated by sliding windows of $250 \mathrm{keV}$ and $1.5 \mathrm{MeV}$.

where $D_{l, i}$ and $M_{l, i}$ are respectively the rates of the data and simulated spectra for a cell $l$ and an energy bin $i$. The terms $\varphi_{i}$ are completely free, but common to all cells, allowing to be independent of any prediction of the energy spectrum. Finally, the nuisance parameters $\alpha$ modelise the systematic uncertainties of the experiment and are constrained by the values of their respective $\sigma$.

The $\Delta \chi^{2}$ test has shown that the data are compatible with the no-oscillation hypothesis with a p-value of $17 \%$. Then the CLs method has been used to draw the exclusion contours at $95 \%$ C.L. shown in figure 4 . The RAA acceptance contour is well covered and especially the best fit point which is excluded at more than $4 \sigma$.

\section{References}

[1] G. MENTION et al., Phys. Rev. D, 83, 073006, 2011, DOI : https://doi .org/10.1103/ PhysRevD. 83.073006. arXiv: https://arxiv.org/pdf/1101.2755.pdf

[2] H. ALMAZAN et al., Phys. Rev. D, 102, 052002, 2020, DOI : https://doi.org/10. 1103/PhysRevD. 102.052002. arXiv : https://arxiv.org/pdf/1912.06582.pdf

[3] H. ALMAZAN et al., J. Phys. G: Nucl. Part. Phys., 48, 075107, 2021, DOI : https://doi . org/10.1088/1361-6471/abd37a, arXiv: https://arxiv.org/pdf/2010.01876.pdf 